\title{
Developmental Differences in Motor Task Integration: A Test of Pascual-Leone's Theory of Constructive Operators
}

\author{
JOHN I. TODOR
}

University of Michigan

\begin{abstract}
This study assessed the ability of Pascual-Leone's Theory of Constructive Operators to predict the minimum age or maturational level at which integration of a motor task could be achieved. Children 5-12 years of age $(n=114)$ performed a discrete motor task requiring a constrained circular movement to be integrated with an unconstrained linear movement to a target. The Theory of Constructive Operators and the principles of constructive cognition were used to generate a model of task performance. Based on the model and in accordance with the theory, it was predicted that 5- to 6-year-old subjects would lack the cognitive capacity ( $M$-capacity) to efficiently integrate this task. An analysis of covariance for age was performed on task parameters reflecting integration (and highest $M$-demand) with movement speed as the covariate. Scheffe contrasts supported the prediction as 5- to 6-year-old subjects were inferior to each of the other age groups $(p<.05)$. Furthermore, no significant differences were found to exist between any of the older age groups.
\end{abstract}

According to Pascual-Leone's Theory of Constructive Operators (Pascual-Leone, 1970, 1976), the maturational limits on cognitive capacity are quantifiable and can account for major developmental changes in information processing observed through childhood. Supportive evidence to date, has been derived largely from the use of Piagetian tasks, or similar "cognitive" tasks. The purpose of this investigation was to test the theory's predictive ability with regards to the performance of a motor task.

In Pascual-Leone's theory, psychological processes are conceived as being hierarchically organized in two interacting systems. The first level is constituted by situational-specific constructs (schemes) which apply on input to categorize or modify it. All schemes become activated or apply

Appreciation is expressed to Juan Pascual-Leone for his assistance in the task analysis. This investigation was supported by a grant from the Office of Research Administration, University of Michigan. Address reprint requests to the author at: Department of Physical Education, University of Michigan, 401 Washtenaw Ave., Ann Arbor, MI 48109. 
according to a cue-function principle, i.e., when the features of reality correspond to the qualitative properties of the scheme it will apply, unless prevented from doing so by some other scheme. As such, schemes represent the perceptual, motor, affective, and cognitive activities available to a given subject.

The second level is constituted by situational-free metaconstructs which apply on the first level schemes (not on the input) to temporarily increase their potential of applying. Although a number of metaconstructs are explicated (see Todor, 1978; Pascual-Leone \& Goodman, Note 1), of particular importance to this paper is the notion of the $M$-operator.

Theoretically defined, the $M$-operator represents a quantification of the individual's cognitive capacity. Conceptually, $M$ may be thought of as a limited amount of mental energy capable of activating or keeping active, items of information (figurative schemes) or plans for action (operative schemes) which are not sufficiently facilitated by other metaconstructs or activated by direct perceptual input. In this sense, there is a similarity between the $M$-operator and the functional properties of mental attention, working memory, and the immediate processor (Kahneman, 1973; Newell \& Simon, 1972; Norman \& Lindsay, 1972).

For children whose cognitive development is normal, $M$-capacity has been demonstrated to increase according to Piaget's substages (Case, 1972a, 1974; Scardamalia, 1977; Toussaint, 1974; DeAviala, Note 2; Parkinson, Note 3).

The left column of Table 1 contains the different values taken by $M$ developmentally. It is convenient to distinguish between two constituents: the constant $e$ and the developmental variable $k$. The constant $e$ represents the $M$-capacity or mental attention necessary to weight or boost the subject's executive scheme (i.e., the psychological organism's representation of task instructions and the corresponding plans for solving it). This quantity is assumed to be invariant across ages.

The developmental variable $k$ corresponds to the maximum additional

TARI.F 1

Predicted $M$-CApacity

\begin{tabular}{clc}
\hline $\begin{array}{c}\text { Predicted } \\
\text { maximum } M \\
(e+k)\end{array}$ & \multicolumn{1}{c}{ Piaget's substages } & $\begin{array}{c}\text { Chronological } \\
\text { age } \\
\text { (years) }\end{array}$ \\
\hline$e+2$ & Last substage of preoperational period & $5-6$ \\
$e+3$ & Low concrete operation & $7-8$ \\
$e+4$ & High concrete operation & $9-10$ \\
$e+5$ & Substage: intro to formal operation & $11-12$ \\
$e+6$ & Low formal operation & $13-14$ \\
$e+7$ & High formal operation & $15-16$ \\
\hline
\end{tabular}


number of discrete items of information or plans which can be activated solely under the direction of the executive. This capacity, together with the executive sophistication of the subject, corresponds to what Piaget labeled the child's "operativity level."

According to the theory, if the task's maximum $M$-demand exceeds the subject's $M$-capacity, then the task cannot be successfully accomplished. Determination of a task's $M$-demand requires a process of rational reconstruction, where the experimenter, using the constructs of the theory, develops a model of the processes which he believes to produce the observed performance. In the case of many tasks, more than one performance strategy may bc used. Thus, care must be taken to specify all alternative strategies and a concerted effort made to identify strategies with the lowest $M$-demand which will permit success. The $M$-demand of a task corresponds to the maximum number of schemes that must be simultaneously boosted by $M$ in order to execute the most efficient strategy. It should be noted that not all schemes involved in a given task require $M$-boosting. The process of generating a model for a task previously has been applied to Piagetian tasks (Case, 1972a, 1972b; PascualLeone \& Smith, 1969). Additionally, a similar sort of approach (without the attempt at quantification) can be found in the work of computer simulators such as Newell and Simon (1972) or investigators interested in the study of artifical intelligence like Minsky (1975) and Papert (1973).

The present study was designed to assess the ability of the Theory of Constructive Operators to predict the minimum age or maturational level at which integration of the components of a motor task could be achieved.

\section{METHOD}

Motor performance was assessed via the performance of a modified Rho task (Fig. 1) (Mendryck, 1960; Todor, 1975). The subject's task was to respond to the light stimulus by moving the crank handle around to the bumper, letting go, and knocking the target down. This movement was to be performed as rapidly as possible. Latencies were recorded for: Reaction Time (RT), the interval between the onset of the light stimulus and the beginning of movement; Circular Time (CT), the time taken to move through the circular phase and to hit the bumper; Pause Time (PT), the length of delay caused by hitting the bumper. This period was initiated by contact of the bumper and terminated when the hand passed over a photoelectric cell positioned immediately in front of the bumper; and Linear Time (LT), the time from the end of the PT to knocking down the target.

\section{Task Analysis}

The following theoretical assumptions were made with respect to the performance of the Rho task. 


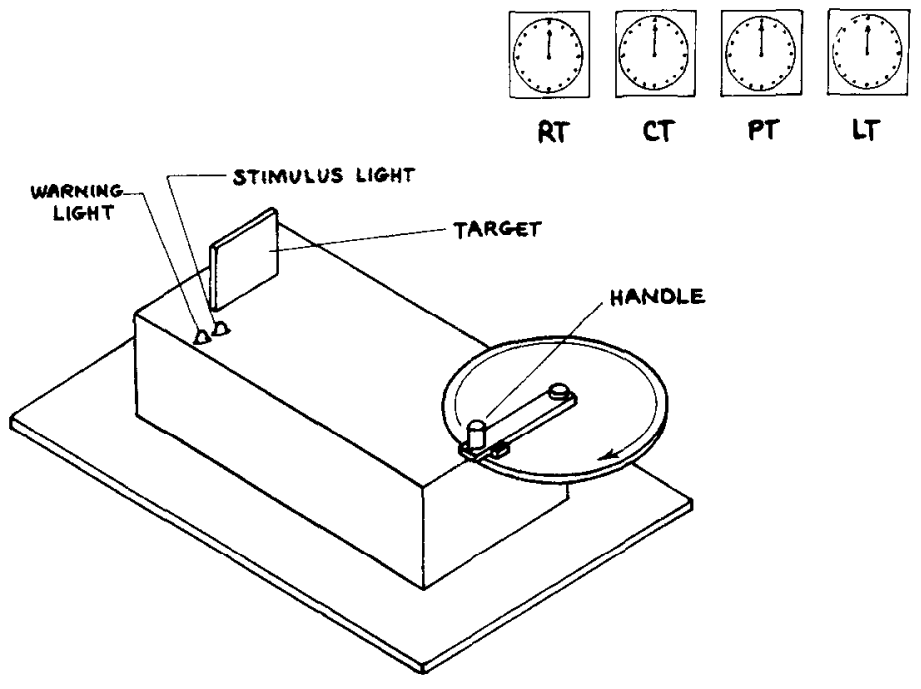

FIG. 1. The Rho task.

Reaction Time Component. Preliminary instruction and practice would insure that subjects had an overlearned scheme in which the activating condition would be the stimulus light onset and the effect a forward PUSH of the handle. $M$-boosting would not be required at this phase.

Circular Movement Component. Since the handle would rotate through a fixed pattern only, the command to PUSH, activated by the light, would suffice if activated until contact with the bumper. $M$-boosting would not be required at this phase (although not pertinent to current predictions, performance of this phase would be facilitated by the metaconstructs C, content learning, and F, field effects-sensorial; see Todor, 1978; Pascual-Leone \& Goodman, Note 1).

Pause Time and Linear Movement Components. The PT and LT components of the task need to be considered together since they would reflect efficient integration of the $\mathrm{CT}$ component with the remainder of the task. It was assumed that the minimum number of schemes necessary for effective integration would be as follows: an operative scheme directing the move to the target; a scheme representing knowledge that the hand is open; and, a scheme representing the location of the target. All three schemes would require $M$-boosting to insure simultaneous co-activation.

Table 2 depicts the strategy necessary for efficient integration of the CT with the PT and LT components. Also depicted are strategies with lower $M$-demands but resulting in less efficient performance. Strategies (2) and (3) denote plans where only two schemes are simultaneously $M$-boosted and the corresponding behavioral consequences. Strategy (4) represents by far the least efficient, where each phase of the task is performed under monitoring of a separate executive scheme. 
TABLE 2

Metasubjective ANAL ysis of Rho TASK INTEgRation

(1) $\epsilon_{\mathrm{l}},{ }^{a} \dot{\psi}$ Move to target,

\# Open hand,

\# Target

(2) $\epsilon_{\mathrm{l}}, \dot{\psi}$ Move to target, \# Open hand

(3) $\epsilon_{\mathrm{I}}, \stackrel{\leftrightarrow}{\psi}$ Move to target, \# Target

(4) $\epsilon_{1}^{1} \quad \epsilon_{1}^{2} \quad \epsilon_{1}^{3}$
(Most effective task integration, Fast PT and LT $M$ demand $=3$

Leads to relatively fast PT but slow LT $M$ demand $=2$

Leads to slow PT and fast LT, Relative to (2) $M$ demand $=2$

Chaining of executives least effective $\epsilon_{\mathrm{I}}^{1}=$ Rotate

$\epsilon_{\mathrm{I}}^{2}=$ Open hand $\epsilon_{\mathrm{I}}^{3}=$ Move to target

Slow PT and LT

${ }^{a}$ Symbols: $\epsilon_{\mathrm{I}}$, executive scheme "Go to target" activated upon contact with bumper;

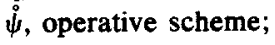

\#, parameters (figurative schemes).

In accordance with the theory, it was predicted that typical 5- to 6-year-old subjects ( $M$-capacity $=2$ units) lack the maturational capacity to effectively integrate this task.

\section{Subjects}

The subjects were 114 children ranging in age from 5 to 12 years. Descriptive statistics are provided in Table 3. Children were drawn from a middle class elementary school after receiving parental consent.

$M$-capacity was estimated by the Compound Stimulus Visual Information Task (CSVI). A detailed description of this task may be found in Pascual-Leone (1970). In this task, each child was taught to elicit a specific motor response (e.g., clap hands, stand up, blink eyes) to a specific visually presented stimulus characteristic (e.g., red, square, X). Once the child demonstrated complete mastery over the cue-response pairs, testing began. In the test condition, each cue card contained from one to eight cues and was presented for a 5 -sec period. To succeed, the child had to retain each cue in immediate memory until the appropriate response had been produced. An individual's score was taken as the mean number of correct responses over the test series.

To perform the Rho Task (Fig. 1), subjects were seated facing the target with the bumper in line with their midline. Prior to test trials, children were required to demonstrate that they understood the task instructions 
TABLE 3

Sample Descriptive Statistics

\begin{tabular}{ccccc}
\hline $\begin{array}{c}\text { Age group } \\
\text { (years) }\end{array}$ & Mean age (months) & & & \\
& & $N$ & Females & Males \\
$5-6$ & $73.53(4.85)$ & 30 & 17 & 13 \\
$7-8$ & $93.93(6.55)$ & 43 & 25 & 18 \\
$9-10$ & $117.58(7.45)$ & 24 & 13 & 11 \\
$11-12$ & $140.41(6.39)$ & 17 & 8 & 9 \\
\multicolumn{1}{l}{ Total } & & 114 & 63 & 51 \\
\hline
\end{tabular}

${ }^{a}$ Standard deviation in parentheses.

and had the required schemes in their repertoire. This was accomplished by having subjects perform the task at a slow speed.

Each trial consisted of a warning light, a variable foreperiod (2-4 sec), and a stimulus light which prompted the movement. Intertrial intervals were approximately $30 \mathrm{sec}$, a sufficient time to reduce fatigue. Thirty consecutive trials were administered.

The CSVI and Rho tasks were individually administered during independent testing sessions separated by at least 1 day.

\section{RESULTS AND DISCUSSION}

Scrutiny of individual CSVI scores revealed four subjects whose $M$-capacity was inaccurately predicted by chronological age. These subjects were 6-year-olds with CSVI values in excess of $1 S D$ of the 5- to 6-year-old group mean, and within $1 S D$ of the 7- to 8-year-old group mean. Since theoretical predictions were made on the bases of $M$-capacity rather than age group, subsequent analyses included these subjects with the 7- to 8-year-olds.

For each task component, the means of the last five trials were taken to represent the final performance on the Rho task. Preliminary evaluation of the data indicated the magnitude of within-group variances on a given task component decreased with increases in age and the corresponding decrease in the observed latencies. To equalize variances all raw data were subjected to a logarithmic transformation prior to subsequent analysis. An analysis of variance was used to compare the RT and CT performances across each age, rather than across $M$-levels. This analysis confirmed an overall decrease in performance time for both task components $(F(7,106)$ $=13.899, p<.0001$ for RT and $F(7,106)=11.048, p<.001$ for CT). Scheffé contrasts for both RT and CT indicated no significant difference to exist between any adjacent ages with no significant differences occurring between subjects 9 years or older. This suggests that performance of these two components increased in a continuous fashion with age, reach- 
ing an apparent ceiling around the age of 9 years. It should be noted that performance of RT and CT components was not predicted to require $M$-boosting. Hence, the relatively continuous improvement observed is not surprising and is likely attributable to increases in strength and/or movement speed per se.

To test the prediction that efficient performance of both PT and LT components required a specified $M$-capacity, a covariance adjustment for CT was necessary. When the task is efficiently integrated the PT + LT components are essentially a follow-through of the circular movement. Consequently, given two subjects with equal integrative ability, the one with the lowest CT latency would have the fastest PT + LT times. Adjustment for differences in CT latencies is especially crucial considering the trend for CT times to decrease with age.

An analysis of covariance, using CT as a covariate, revealed an overall age group difference in PT + LT latencies $(F(3,109)=22.133, p<.0001)$. (Table 4.) Scheffé contrasts indicated the 5- to 6-year-old group to be significantly inferior when compared to each of the other groups $(p<.05)$. Furthermore, no significant differences were found to exist between any of the three older age groups. The increased time taken by the 5- to 6-year-old subjects supports the prediction that due to their available $M$-capacity, they could not effectively integrate this task.

The results of this study indicate the Theory of Constructive Operators has considerable predictive and explanatory ability with respect to the performance of motor tasks. As predicted by the model, and demonstrated, the typical 5- to 6-year-old subject could not effectively coordinate the components of the task, a factor differentiating them from all older subjects. This stratification was not evident in the reaction time and circular movement components where a continuous improvement in performance was observed across ages to an apparent ceiling. Performance of these components imposed limited demands on cognitive capacity and may be influenced by factors such as muscular strength and movement speed per se.

TABLE 4

Means, Adjusted Means, and Scheffé Contrasts for PT + LT

\begin{tabular}{ccccccc}
\hline & & & \multicolumn{3}{c}{ Scheffé contrasts } \\
\cline { 4 - 6 } Group & $\bar{X}$ & Adjusted $\bar{X}$ & $7-8$ & $9-10$ & $11-12$ \\
\hline $5-6$ & 343.78 & 325.33 & $*$ & $*$ & $*$ \\
$7-8$ & 260.09 & 247.54 & - & ns & ns \\
$9-10$ & 226.80 & 246.63 & & - & ns \\
$11-12$ & 173.72 & 208.64 & & & & - \\
\hline
\end{tabular}

$* p<.05$. 
In contrast to the Theory of Constructive Operators, it could be argued that the inferior performance of 5- to 6-year-old subjects reflected poor executive/control structures which would improve with extended practice. An earlier study evaluating the effects of practicing the Rho task to an essentially asymptotic level, indicates this not to be the case (Todor, 1975).

In the earlier study the CT vs LT (LT reflected both PT \& LT) correlations of three disparate age groups were compared across six blocks of practice trials (five trials per block). Due to the nature of the Rho task, the performance of the LT component (as defined in the earlier study) should be positively related to the performance of the circular component, since when the task is performed rapidly, the former should function as a continuation or follow-through of the latter. Other factors such as a relatively slow CT followed by a well-integrated LT would tend to decrease the relationship, therefore, one would not expect the correlation to be high.

For 18-year-old subjects who required few practice trials to reach an asymptotic level of performance, the block-by-block correlations fluctuated slightly around $r=.40$. For 11-year-old subjects whose performance reached the 18-year-old level by block 6 , correlations between components increased across blocks from approximately $r=.20$ to $r=$ .40 . This suggests that practice led to an increased integration by these subjects.

In contrast, the CT vs LT correlations for the 6-year-old subjects tended to decrease with practice (block $1 r=.36$, block $6 r=.08$ ). These subjects were apparently unable to coordinate the two components into a well-integrated performance, a fact predictable by their available $M$-capacity and verified in the current study. It should be noted that through practice the 6-year-old subjects improved their performance of both task components. This suggests that their performance strategy led to performing the components as two independent tasks.

Based on the findings of the current and earlier Rho task studies, it seems reasonable to conclude that if a child lacks the necessary developmental capacity to integrate all task components, he adopts a compensatory performance strategy. In the case of the Rho task, it appears that 5- to 6-year-old subjects reduce the task demands to comply with their available cognitive capacity by essentially performing two independent movements.

\section{REFERENCES}

Case, R. Validation of a neo-Piagetian capacity construct. Journal of Experimental Child Psychology, 1972a, 14, 287-302. 
Case, R. Learning and development: A neo-Piagetian interpretation. Human Development, $1972 b, 15,339-358$.

Case, R. Structures and strictures: Some functional limitations on the course of cognitive growth. Cognitive Psychology, 1974, 6, 544-573.

Kahneman, D. Attention and effort. Englewood Cliffs, N.J.: Prentice-Hall, 1973.

Mendryck, S. Reaction time, movement time and task specificity relationships at ages twelve, twenty-two and forty-eight years. Research Quarterly, 1960, 31, 156-162.

Minsky, M. A framework for representing knowledge. In P. Winston (Ed.), The psychology of computer vision. New York: McGraw-Hill, 1975.

Newell, A., \& Simon, H. A. Human problem solving. New York: Prentice-Hall, 1972.

Norman, D. A., \& Lindsay, P. H. Human information processing: An introduction to psychology. New York: Academic Press, 1972.

Papert, S. Theory of knowledge and complexity. In G. J. Dalenoort (Ed.), Process models for psychology. Netherlands: Rotterdam Univ. Press, 1973.

Pascual-Leone, J. A mathematical model for the transition rule in Piaget's developmental stages. Acta Psychologica, 1970, 32, 301-345.

Pascual-Leone, J. Metasubjective problems of constructive cognition: Forms of knowing and their psychological mechanism. Canadian Psychological Review, 1976, 17, 110 125.

Pascual-Leone, J., \& Smith, J. The encoding and decoding of symbols by children. Journal of Experimental Child Psychology, 1969, 8, 328-355.

Scardamalia, M. Information processing capacity and the problem of horizontal décalage: A demonstration using combinatorial reasoning tasks. Child Development, 1977, 48, 28-37.

Todor, J. I. Age differences in integration of components of a motor task. Perceptual and Motor Skills, 1975, 41, 211-215.

Todor, J. I. A neo-Piagetian theory of constructive operators: application to perceptualmotor development and learning. In D. M. Landers and R. W. Christian (Eds.), Psychology of motor behavior and sport. Urbana, Ill.: Human Kinetics Publishers, 1978. Pp. 507-521.

Toussaint, N. A. An analysis of synchrony between conrete-operational tasks in terms of structural and performance demands. Child Development, 1974, 45, 992-1002.

\section{REFERENCE NOTES}

1. Pascual-Leone, J., and Goodman, D. Intelligence and experience: A neo-Piagetian approach. Unpublished manuscript, York Univeristy, 1977.

2. DeAviala, E. A. Children's transformation of visual information according to nonverbal syntactical rules. Unpublished doctoral dissertation, York University, 1974.

3. Parkinson, G. M. The recognition of messages from visual compound stimuli: a test of a quantitative developmental model. Unpublished master thesis, York University, 1969.

Received: May 1, 1978; Revised: July 11, 1978, January 22, 1979. 